\title{
Magnetostrictive actuator with differential displacement amplification mechanism
}

\author{
Quanguo Lu*, Qin Nie**, Xiaoyang Jiang***, Qinghua Cao****, Dingfang Chen***** \\ *Institute of Micro/Nano Actuation and Control, Nanchang Institute of Technology, Nanchang, China, 330099 , \\ E-mail:Luqg2010@126.com \\ **Institute of Micro/Nano Actuation and Control, Nanchang Institute of Technology, Nanchang, China, 330099, \\ E-mail:1134598717@qq.com \\ ***Institute of Intelligent Manufacturing and Control, Wuhan University of Technology, Wuhan, China, 430063, \\ E-mail:18575535576@163.com \\ ****Institute of Micro/Nano Actuation and Control, Nanchang Institute of Technology, Nanchang, China, 330099, \\ E-mail:QH9863@126.com \\ *****Institute of Intelligent Manufacturing and Control, Wuhan University of Technology, Wuhan, China, 430063, \\ E-mail: cadcs@126.com
}

cross ${ }^{\text {ref }}$ http://dx.doi.org/10.5755/j01.mech.22.4.16166

\section{Introduction}

The giant magnetostrictive material (GMM) has been developed in recent years as a new type of functional material [1]. It has large strain, high energy density, a large output force, and a high response [2]. The actuators, which use GMM as a drive resource (GMA), are very precise and have a rapid response speed, but the output displacement is only on the micron level [3]. Therefore, this type of actuator cannot meet the demands of large output displacement [4]. To remove this restriction, a GMM with a flexure hinge mechanism was designed. This actuator has a compact structure, large displacement, clear transmission relation, no transmission idle stroke and no friction. It is especially suitable for use with a precision drive structure [5].

In order to make the output displacement large and precise, Cuihong Li of Tsinghua University [6] used GMA as the driving source and piezoelectric ceramic as a clamp. Based on the creeping principle, the piezoelectric ceramic was embedded in the axial symmetric flexible hinge, however, the entire structure was complex and it was difficult to determine the peristaltic step. Yang Bintang [7] of Shanghai Jiao Tong University devised a type of GMA which had large stroke and high resolution. The design was based on the telescopic driving principle of GMM and the flexible hinge amplification mechanism. However, it required a large number of actuators and was expensive to design. Furthermore, the design did not optimize the structure of the actuator. Japan's Ibaraki University EDA Hong [8], in conjunction with the Toshiba Co., Kobavashi designed a GMA whose positioning accuracy could reach micron level, and has been successfully applied to the GMA for a micro feeding device with a large optical diamond lathe [9].

For this study, a GMA was created using differential displacement amplification, giant magnetostriction material and the flexible hinge amplification mechanism. Based on the fundamental characteristics of magnetostrictive material, this study analyzed the actuator prototype and created a new design for GMA including a drive coil, preloading mechanism and displacement amplification mechanism. This research primarily analyzed the influence of the input current and pre-pressure on the output displacement
[10]. Moreover, the reasonable matching problem of displacement amplification mechanisms and the GMA was analyzed [11].

\section{Materials and methods}

2.1. Design of differential amplifying magnetostrivtive actuator

As shown in Fig. 1, the structure of the differential amplification micro actuator was primarily composed of GMA and a displacement amplification mechanism. Micro displacement produced by the actuator was transformed into large displacement in the output end of the structure through the transmission in the amplification mechanism.

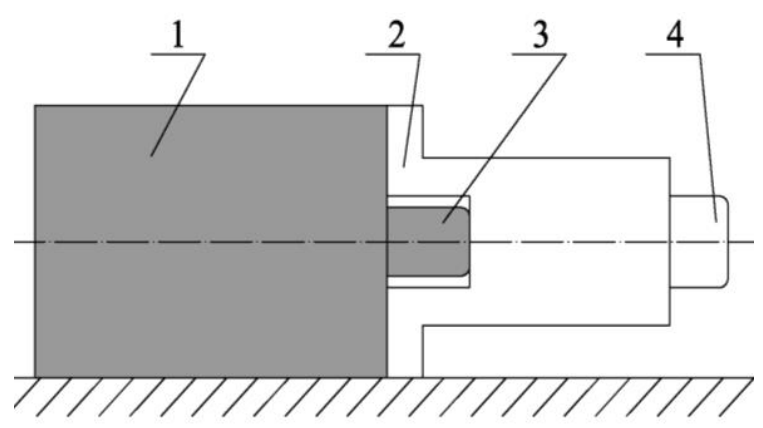

Fig. 1 Structure diagram of GMA with large displacement: 1 -GMA; 2 -Displacement amplification mechanism; 3 -Output shaft; 4 -Output of amplification mechanism

The output shaft of the micro actuator and the output end of the displacement amplification mechanism were on the same axis, and moved in the same direction so as to ensure the stability and same-phase of the displacement amplification.

\subsection{Design of micro GMA}

GMA is a type of magnetic-mechanical coupling 
converter, and its design relates to the selection of magnetostrictive material and the devise of the coil, the magnetic circuit and the preloading mechanism. In this article, as shown in Figure 2, the structure of the actuator is composed of a GMM rod, sleeve, coil, coil skeleton, output shaft, commutator end bearing bracket, drive end bearing bracket, shell, preloading nut, preloading spring, linear bearing, as well as other components. The GMM rod, output shaft, shell and commutator end bearing bracket consists of a closed magnetic circuit, therefore reducing magnetic leakage and improving the strength of the magnetic field. The output shaft, drive end bearing bracket, preloading spring and preloading nut consists of a preloading mechanism. By applying the appropriate preloading pressure, the GMM rod exhibits a large axial strain while simultaneously increasing the output displacement and force. The GMM rod is made of brittle material, which can resist pressure, but not tension. Therefore, imposing a certain preloading stress can make the GMM rod work in the compression state.

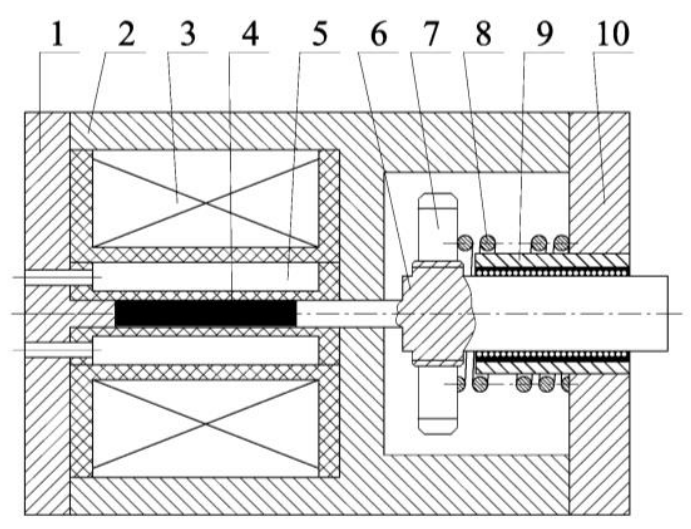

Fig. 2 Structure of GMA: 1 -Commutator end bearing bracket; 2-Sleeve; 3-Coil; 4-GMM rod; 5 - Coilformer; 6 - Output shaft; 7 - Linear bearing; 8- Preload nut; 9 - Preload spring; 10 - Shell

\subsection{Design of preloading mechanism}

In order to fully utilize the magnetostrictive properties of GMM, this research is based on a design that allows the preloading mechanism to put suitable pre-pressure onto the GMM rod [12]. If the pre-pressure applied is either too large or too small, the magnetostrictive effect of the GMM rod will be hindered. Therefore, the selection of the amount of the pre-pressure should be based on the optimal energy conversion efficiency of the differential amplifying micro actuator; which for this research, $7 \sim 10 \mathrm{MPa}$ was chosen.

Pre-pressure can be applied in various models, usually by devices such as a cylindrical helical spring, disk spring, and elastic plates. The device can be relatively simplified by using a combination of a cylindrical spiral spring with a preloading nut, so it can conveniently adjust the prepressure and ensure the direction of pre-pressure in parallel with the axis of the GMM rod. The pre-pressure, if evenly distributed, aids the GMM rod from bending.

For this research, the cylindrical spiral spring and displacement amplification mechanism was used to apply axial pre-pressure. This method was used because it can adjust the compression degree of the cylindrical helical spring by rotating preloading nut, as well as adjust the deformation of the displacement amplification mechanism under an initial state. Furthermore, the output shaft of the displacement amplification mechanism can simultaneously apply prepressure to the GMM rod by counterforce.

\subsection{Design of displacement amplification mechanism}

The displacement amplification mechanism is the core component of the differential amplifying micro actuator. The main function of the displacement amplification mechanism is to enlarge the output displacement to a certain extent. Its performance determines the performance of the differential amplifying micro actuator. The realization of micro displacement amplification has many forms, such as lever amplification, gear combination, ellipse amplification, and hydraulic amplification [13]. For this study, differential lever amplification combined with the flexible hinge structure was used. A principle diagram of differential lever amplification is shown in Fig. 3. According to the different input directions, differential lever amplification can be divided into two types, synthetic drive and reverse drive. The amplification effect of these two types is equal. In order to acquire a rational arrangement of structure, synthetic drive was used.
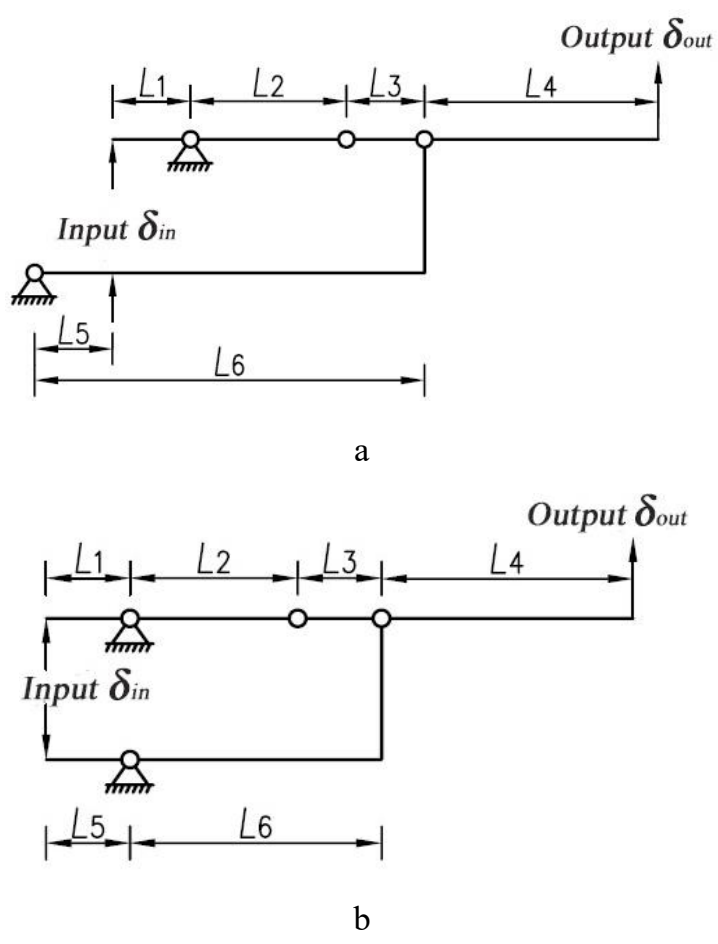

Fig. 3 Principle diagram of differential lever: a- synthetic drive; b - reverse drive

Ignoring the elastic deformation of the lever and the resistance of the hinge, this study assumed that the lever was a rigid body, the hinge was an ideal hinge, and the input displacement was $\delta_{i n}$. According to the principle of lever, the output displacement was:

$$
\delta_{\text {out }}=\left[\frac{L_{4}}{L_{3}}\left(\frac{L_{2}}{L_{1}}+\frac{L_{6}}{L_{5}}\right)+\frac{L_{6}}{L_{5}}\right] \delta_{i n} .
$$

The definition of magnification was the ratio of 
output displacement and input displacement. It is an important index to measure the amplification ability of the amplification mechanism. In this study, magnification of the differential amplification mechanism was:

$$
A=\frac{\delta_{\text {out }}}{\delta_{\text {in }}}=\left[\frac{L_{4}}{L_{3}}\left(\frac{L_{2}}{L_{1}}+\frac{L_{6}}{L_{5}}\right)+\frac{L_{6}}{L_{5}}\right] .
$$

The displacement amplification mechanism is shown in Fig. 4. The output displacement of the GMM rod acted on the input end of the displacement amplification mechanism, and the input displacement was located at the output terminal after amplified in the displacement amplification mechanism. The realization of movement transmission relied on the micro rotational deformation of the flexible hinge, therefore, the flexible hinge needed to have good elastic recovery ability. Researchers selected $60 \mathrm{Si} 2 \mathrm{Mn}$ as the material of the displacement amplification mechanism [13]. As a result, the displacement amplification mechanism had the advantage of a simple structure, compact use of space, no friction, no abrasion, no clearance, no backlash, strong recovery ability and high motion sensitivity.

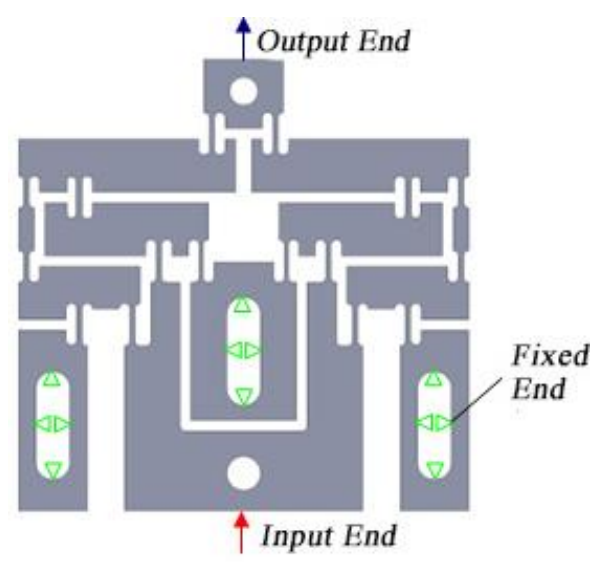

Fig. 4 Structure of displacement amplification mechanism



Fig. 5 Assemble diagram of displacement amplification mechanism

Fixed installation of the displacement amplification mechanism was a key problem. If there were three fixed ends that were not placed solidly, the displacement would be lost during the transfer process. Also, there would be a decrease in performance of the displacement amplification mechanism. In order to avoid installation problems, the assembly structure of displacement amplification mechanism, a splint was made in a convex shape and was used for clamping the three fixed ends of the displacement amplification mechanism (Fig. 5). An abutment was placed on the front end and was used to hold the splint. High strength bolts connected the splint and the abutment, therefore, the displacement amplification mechanism had been fixed by the friction of contact surfaces.

\section{Experiment}

On the basis of design analysis, researchers trialproduced the prototype of the differential amplification micro actuator and a process performance test was executed.

\subsection{Introduction of experimental system}

The experimental system of the differential amplification micro actuator was composed of the micro actuator, vibration isolation platform, temperature controller, constant current source, displacement measuring system, constant temperature water cooling system and magnetic field detection system.

The experimental system adopted the PC machine as the controller. The digital signal sent by the PC machine was converted into an analog voltage signal through PCI$8333 \mathrm{D} / \mathrm{A}$. Then, the voltage signal was used as the input into the controllable constant current source. The constant current source output corresponding currents to the drive coil, and the coil produced a magnetic field. In this magnetic field, the GMM rod produced magnetostrictive deformation. The deformation output was located at the output end of the displacement amplification mechanism. After being amplified, the output displacement signal was sent to the PC through use of a LVDT micrometer. Temperature controller measured the temperature of cooling water in the constant temperature water tank through the temperature sensor. The cooling water, whose function was to dissipate the heat generated by the drive coil, flowed in a circular motion between the water cooling cavity and the constant temperature water tank, so as to ensure the GMM rod would work in the state of constant temperature. A flux meter was used for measuring the driving magnetic strength distributed in the GMM rod. In order to reduce the effect of the external environment on the data measurement, the actuator was placed on the isolation platform.

\subsection{Experiment on the characteristics of output displacement}

In order to measure the output displacement of the amplification mechanism and determine the balance position, the displacement sensor was located on the output end of the displacement amplification mechanism, and the output displacement was measured using a micrometer LVDT. After pre-pressure was applied to the GMM rod, the displacement amplification mechanism produced deformation due to the reaction force. The output end produced a certain displacement, which was defined as the initial displacement. 
As a result, the pre-pressure was weighed by the initial displacement and the greater the initial displacement, the greater the pre-pressure. To accurately measure the initial displacement, the determination of the equilibrium position was key, therefore, the equilibrium position was determined by the LVDT micrometer.

\section{Results analysis}

The initial displacement was $234 \mathrm{~mm}$ and the input current was $0 \sim 4$ A to the differential amplification micro actuator. The result of the output displacement is shown in Fig. 6. The output displacement increased as the current increased from $0 \sim 4 \mathrm{~A}$. On the contrary, the output displacement decreased as the current decreased from $4 \mathrm{~A} \sim 0$. However, in the return process, the output displacement displayed a hysteresis phenomenon, and could not return to the lift stroke curve along with the current decrease. The phenomenon is termed the hysteresis effect.

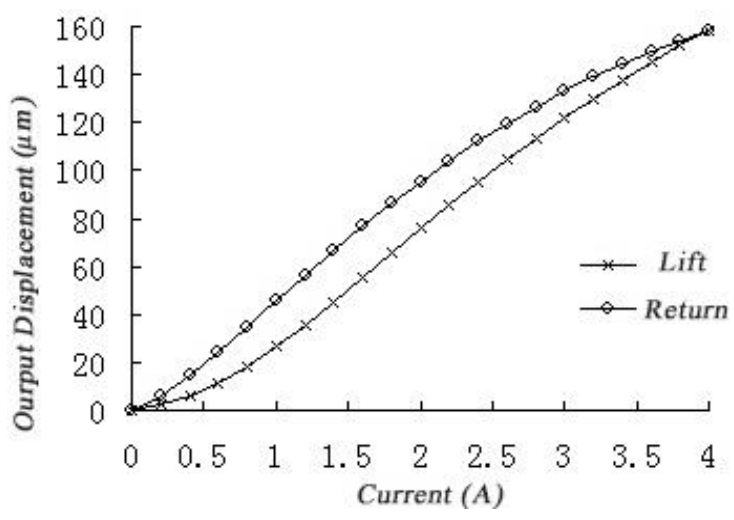

Fig. 6 Hysteresis curve of 4 A current

$$
e=\frac{\max \left|y_{2 i}-y_{1 i}\right|}{\max \left(y_{1}\right)} \times 100 \%
$$

Hysteresis can be calculated using the equation shown above. In which, $y_{1}$ represents output displacement in the lift stroke, $y_{1 i}$ represents output displacement in the lift stroke when the corresponding current is $I, y_{2 i}$ represents output displacement in the return stroke when the corresponding current is $I$.

In the situation of current strength changing from $0 \sim 4 \mathrm{~A} \sim 0$, and when the input current is $1.4 \mathrm{~A}$, the difference of output displacement between return stroke and lift stroke reaches the maximum, and $\max \left|y_{2 i}-y_{1 i}\right|=2.15 \mu \mathrm{m}, \max \left(y_{1}\right)=158.4 \mu \mathrm{m}$, and calculated by Eq. (3), the hysteresis $e=13.35 \%$.

The hysteresis is different under the effect of different electric current strength. When the current changing from $0 \sim 1 \mathrm{~A} \sim 0,0 \sim 2 \mathrm{~A} \sim 0,0 \sim 3 \mathrm{~A} \sim 0$, $0 \sim 4 \mathrm{~A} \sim 0$, the corresponding hysteresis is respectively $13.35 \%, 15.76 \%, 19.22 \%, 20.6 \%$, the greater the current is, the more serious the hysteresis phenomenon is. As can be seen from Fig. 7, lift curve is basically coincident together, while the return curve is different for the reason of hysteresis phenomenon.

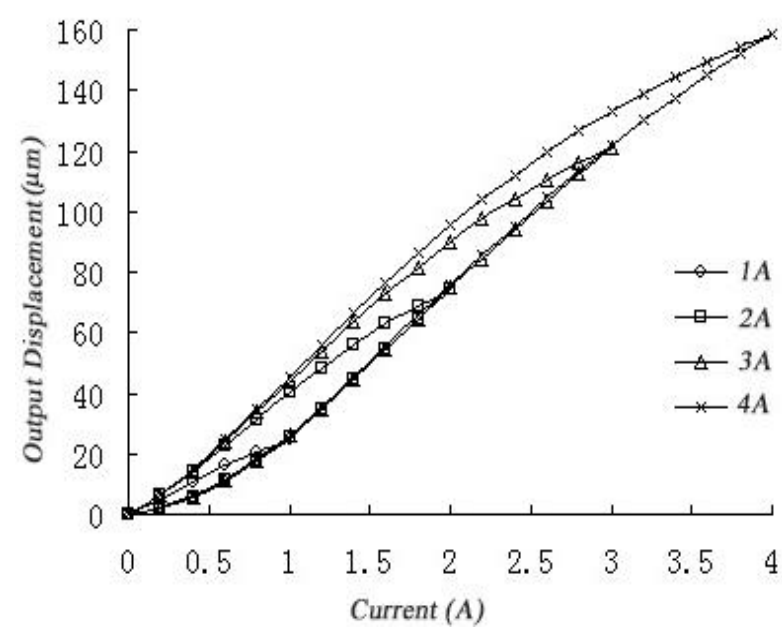

Fig. 7 Hysteresis curve of different current

The displacement amplification mechanism, whose amplification ability determines the maximum output displacement, was the core component of the differential amplification micro actuator. The magnification was used as an index for measuring amplification ability. This experiment was designed to indirectly test the magnification of the displacement amplification mechanism by measuring the input displacement and output displacement.

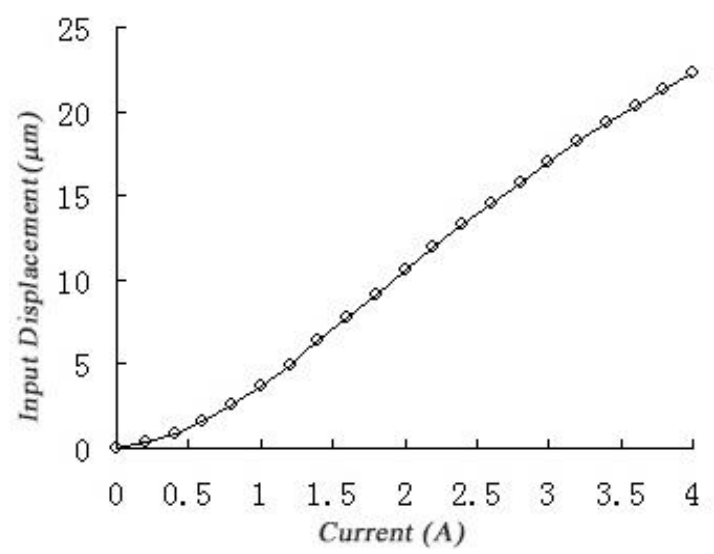

$\mathrm{a}$

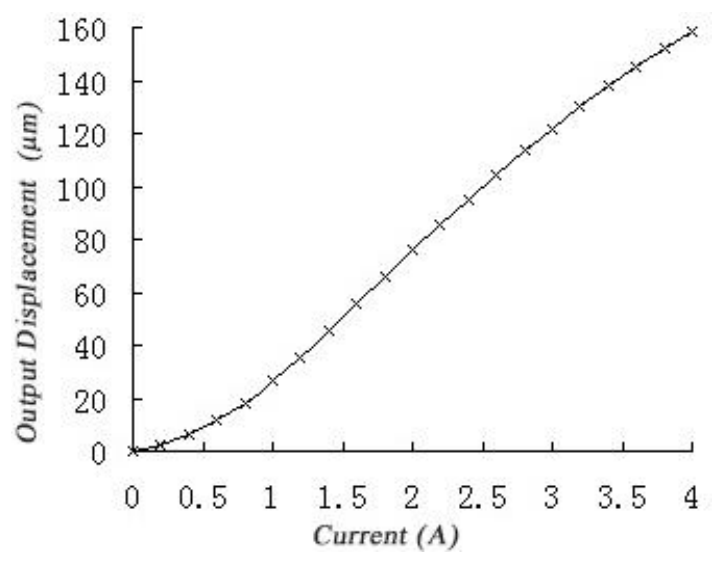

$\mathrm{b}$

Fig. 8 Relationship of displacement and current: a - input displacement and current; b - output displacement and current

The GMM rod produced magnetostrictive defor- 
mation when the input current to the differential amplification micro actuator was $0 \sim 4 \mathrm{~A}$. The relationship between current and input displacement is shown in Fig. 8, a. The input displacement increased with the increasing current, and under $1 \sim 4$ A current strength, the linearity degree of relationship curve was high enough.

The relationship between the current and the output displacement is shown in Fig. 8, b. The output displacement increased with the increasing current, and when current strength was in the range of $1 \sim 4 \mathrm{~A}$, the linearity degree of relationship curve was high enough.

The linearity degree of output displacement and input displacement were both high enough, therefore could be determined as a linear relationship, with the magnification of displacement amplification mechanism being 7.14.

As it can be seen from the Table 1, the bridge amplification is characterized by a high amplify magnification, but is limited by the fact that the input displacement and output displacement are in different directions. The magnification of the lever amplification mechanism is large enough, but the displacement loss becomes larger when there is a load. The eight rod symmetric linkage has a larger amplify magnification compared to the lever amplification, but the structure is large and complicated. Differential amplification can obtain a larger displacement output with the relatively small structure; however, the magnification is not ideal. In this magnetostrictive actuator, the differential principle and the lever principle are combined to achieve the largest possible displacement magnification, resulting in the realization of the synthetic drive, small displacement loss, small counter force and compact structure.

Table 1

Comparison of displacement amplification mechanisms

\begin{tabular}{|c|c|c|c|c|}
\hline No. & Name & Affiliation or Author & $\begin{array}{c}\text { Amplify } \\
\text { Principle }\end{array}$ & $\begin{array}{c}\text { Amplify } \\
\text { Magnifica- } \\
\text { tion }\end{array}$ \\
\hline 1 & $\begin{array}{c}\text { Micro-displacement Amplifier for } \\
\text { GMA [14] }\end{array}$ & $\begin{array}{c}\text { Northwestern Polytechnical } \\
\text { University }\end{array}$ & Lever Principle & 3.8 \\
\hline 2 & $\begin{array}{c}\text { Micro-displacement Amplifier for } \\
\text { Piezoelectric Bimorph [15] }\end{array}$ & $\begin{array}{c}\text { Henan Coal Scientific Re- } \\
\text { search Institute Co., Ltd. }\end{array}$ & Eyght Rod & 6.2 \\
\hline 3 & $\begin{array}{c}\text { Mechanically amplified large } \\
\text { Displacement Piezoelectric Actu- }\end{array}$ & $\begin{array}{c}\text { Juuti J, Kordas K and Lon- } \\
\text { nakko R }\end{array}$ & $\begin{array}{c}\text { Bridge Amplifica- } \\
\text { tion }\end{array}$ & $16 \sim 20$ \\
\hline 4 & $\begin{array}{c}\text { Flexible Hinge Displacement } \\
\text { Amplification Mechanism [17] }\end{array}$ & $\begin{array}{c}\text { China Shipbuilding Industry } \\
\text { Corporation(CSIS) }\end{array}$ & $\begin{array}{c}\text { Differential Ampli- } \\
\text { fication }\end{array}$ & 1.8 \\
\hline 5 & $\begin{array}{c}\text { Differential Displacement Ampli- } \\
\text { fication Mechanism }\end{array}$ & This article & $\begin{array}{c}\text { Differential and } \\
\text { Lever Principle }\end{array}$ & 7.14 \\
\hline
\end{tabular}

\section{Conclusions}

This study designed a new type of differential amplification micro actuator based on the use of giant magnetostrictive material and flexible hinges. The maximum displacement can reach up to $158.4 \mu \mathrm{m}$, and the magnification can reach up to 7.14 . This experiment not only enlarges the output displacement of the micro actuator, but also improves its performance, and therefore leading to the potential of broad applications. Future research for this study can include ways to optimize the structure of the displacement amplifier to make it more precise and compact. Also, research on the characteristics of micro-displacement amplification actuator under the load action can be analyzed.

\section{Acknowledgments}

The authors would like to acknowledge the financial support by the National Natural Science Foundation of China (Grant No.51165035). Science and Technology Fund of Jiangxi Province of Higher Education (KJLD14094), Youth Science Fund of Jiangxi Province (20133BAB21004), and the Young scientist cultivation plan of Jiangxi Province (20112BCB23025).

\section{References}

1. Liang, S.; Leon, M.H. 2013. Nonlinear model for Galfenol cantilevered unimorphs considering full magneto elastic coupling, Journal of Intelligent Material Systems and Structures 12(10): 1-17.

2. Wang, F.J.; Jia, Z.Y.; Liu, W. 2011. Calculation of magnetostrictive coefficient of composite chin film and structure optimization of cantilever, Opt. Precision Eng 19(8): 1832-1838. http://dx.doi.org/10.3788/OPE.20111908.1832.

3. Li, X.X.; Wang, W.; Chen, Z.C. 2010. Generalized predictive-multimode PID control for giant magnetostrictive actuators, Opt. Precision Eng 18(2): 412-420.

4. Zhang, L.; Wu, Y.J.; Liu, X.L. 2012. Linearity hysteresis model of giant matnetostrictive components for non-cylindrical hole precision machining, Opt. Precision Eng 20(2): 87-295.

5. Cao, Q.H.; Lu, Q.G.; Xi, J.M. 2011. Modeling and performance analysis of giant magnetostrictive microgripper with flexure hinge, 2011 IFIP Advances in Information and Communication Technology, Nanchang, P.R. China: 1029-1038.

6. Li, C.H.; Ye, Z.S.; Meng, Y.G. 2005. Linear inchworm 
mechanism based on giant magnetostrictive and piezoelectric materials, Journal of Tsinghua University 45(8): 1055-1057.

7. Yang, B.T.; Zhao, Y.; Peng, Z.K. 2013. Real-time compensation control of hysteresis based on Prandtl-Ishlinskii operator for GMA, Opt. Precision Eng 21(1): 125-130.

8. Rezaeealam, B.; Ueno, T.; Yamada, S. 2012. Finite Element Analysis of Galfenol Unimorph Vibration Energy Harvester, IEEE Transactions on Magnetics 48(11): 3977-3980. http://dx.doi.org/10.1109/TMAG.2012.2202273.

9. Davino, D.; Giustiniani, A.; Visone, C. 2012. Effects of hysteresis and eddy currents in magnetostrictive harvesting devices, Physica B 407: 1433-1437. http://dx.doi.org/10.1016/j.physb.2011.07.038.

10. Jiang, X.Y. 2011. Design and research of micro-actuator with large displacement based on gmm and flexible hinge. Wuhan University of Technology.

11. Karunanidhi, S.; Singaperumal, M. 2010. Design, analysis and simulation of magnetostrictive actuator and its application to high dynamic servo valve, Sensors and Actuators A 157: 185-197.

http://dx.doi.org/10.1016/j.sna.2009.11.014

12. Tao, S.A.; Bai, H.B.; Hou, J.F. 2009. Effect of preliminary pressure on the displacement and hysteresis of the piezoelectric actuators, Ordnance Material Science and Engineering 32(2): 29-32.

13. Pan, J.Z.; Ren, R.M.; Guo, L.B. 2010. Effect of quenching on microstructure and mechanical properties of $60 \mathrm{Si} 2 \mathrm{Mn}$ steel, Material \& Heat Treatment (22): 146148.

14. Sun, Z.L.; Zhao, M.Y.; Yin, Z.D. 2012. Designing micro-displacement amplifier for giant magnetostrictive actuator, Mechanical Science and Technology for Aerospace Engineering 7(31): 1047-1050.

15. zhang, b.; li, s.j.; gong, y.j. 2011. research on mechanism of micro-displacement magnifying of piezoelectric bimorph, Heat Treatment Technology and Equipment (02): 42-45.

16. Juuti, J.; Kordas, K.; Lonnakko, R. 2005. Mechanically amplified large displacement piezoelectric actuators, Sensors and Actuators A (120): 225- 231. http://dx.doi.org/10.1016/j.sna.2004.11.016.

17. Wang, S.M.; Yun, X.; Chen, J.Z. 2011. Design and analysis on micro displacement magnifying mechanism with flexible hinges, Hydraulics Pneumatics \& Seals (01): 17-20.
Quanguo Lu, Qin Nie, Xiaoyang Jiang, Qinghua Cao, Dingfang Chen

\section{MAGNETOSTRICTIVE ACTUATORWITH DIFFERENTIAL DISPLACEMENT AMPLIFICATION MECHANISM}

S u m m a r y

In light of the contradiction between stroke and accuracy of current actuators, a high precision, large displacement actuator was designed. Rare earth giant magnetostrictive material was used as the driving source and the displacement differential amplification was based on a flexure hinge. A magnetic circuit and driving magnetic field was designed with the characteristics of magnetostrictive drive. The axial pre-pressure of the magnetostrictive rod was supported by a cylindrical helical spring and a displacement amplification mechanism. A new type of differential amplification device was projected by the flexible hinge. The convex desktop splint fixed displacement amplification mechanism was adopted to enlarge the magnetostrictive's small displacement. After the actuator prototype was manufactured and the test platform was established, the output characteristics and amplification mechanism performance were analyzed. Results showed that under the condition of 0 to 4 input current, the maximum displacement was $158.4 \mu \mathrm{m}$ and the magnification was 7.14.

Keywords: actuator, rare earth, flexure hinge, differential, displacement amplifier.

Received September 15, 2015 Accepted July 04, 2016 